\title{
CANONICAL DOMAINS ON RIEMANN SURFACES
}

\author{
BERNARD MASKIT
}

(Communicated by Irwin Kra)

\begin{abstract}
Let $S$ be a Riemann surface of genus $g>0$, and of finite topological type. Then $S$ can be uniquely realized as a closed Riemann surface from which a finite number of disjoint points and closed circular discs have been removed. As a corollary, we obtain that the moduli space of surfaces of genus $g$ with one hole is a topological product of the moduli space of surfaces of genus $g$ with one puncture and an interval.
\end{abstract}

It is well known that every planar Riemann surface can be uniquely represented as a slit domain in any one of several classes of such domains. Also, every topologically finite planar Riemann surface can be uniquely represented as a circle domain on the sphere. Some results along these lines are also known for surfaces of higher genus. It was shown by Grötzsch [G] that every topologically finite surface of genus 1 can be represented as a slit domain on a torus; see also Royden [R]. It was shown by Strebel [S] and Jost (unpublished) that every topologically finite surface of genus 1 can be uniquely represented as a circle domain on a torus. It was shown by Haas $[\mathrm{H}]$ that every topologically finite Riemann surface can be represented as a circle domain on a closed Riemann surface of the same genus. In this paper, we extend these results to show that Haas's circle representation is unique for every genus. We then give an application of our result to Teichmüller theory, and show that the moduli space of Riemann surfaces of finite genus $g>0$, with one hole, is topologically equivalent to the product of an interval and the moduli space of Riemann surfaces of genus $g$ with one puncture.

\section{Circle domains on Riemann surfaces}

Let $\bar{S}$ be a closed Riemann surface of genus $g>0$. Then there is a canonical metric on $\bar{S}$ defined as follows. If $g>1$, there is an essentially unique Fuchsian group $F$, acting on the upper half-plane, $\mathbf{H}^{2}$, so that $\bar{S}$ is conformally equivalent to $\mathbf{H}^{2} / F$. We endow $\mathbf{H}^{2}$ with its usual Poincaré, or hyperbolic, metric $d s=|d z| / \operatorname{Im}(z)$, and we call the projection of this metric to $\bar{S}$ the canonical metric.

Received by the editors April 11, 1988 and, in revised form, October 12, 1988.

1980 Mathematics Subject Classification (1985 Revision). Primary 30F10, 32G15.

Research supported in part by NSF Grant \#8701774. 
If $\bar{S}$ has genus 1 , then we can represent $\bar{S}$ as $\mathrm{C} / F$, where $F$ is a fixed point free group of Euclidean motions. Every such motion is a translation; we normalize $F$ so that it has unit minimal translation length. It is easy to see that this normalization is equivalent to requiring that $F$ be generated by $z \rightarrow z+1$, and $z \rightarrow z+\tau$, where $|\tau| \geq 1$, and $|\operatorname{Re}(\tau)| \leq \frac{1}{2}$. We use this normalization for $F$, and call the projection of the Euclidean metric to $\bar{S}$ the canonical metric on $\bar{S}$.

Suppose $\bar{S}$ is a closed Riemann surface of genus $g>0$, and suppose $S$ is a subsurface of $\bar{S}$ where $\bar{S}-S$ has finitely many components, each of which is either a point or a closed circular disc in the canonical metric on $\bar{S}$; then we say that $S$ is a circle domain on $\bar{S}$.

A Riemann surface $S$ is topologically finite if it is homeomorphic to the interior of a compact (orientable) 2-manifold $\bar{S}$; this definition is equivalent to the statement that $S$ can be topologically embedded in a closed surface $\bar{S}$, where $\bar{S}-S$ has finitely many components. Each boundary component of a topologically finite Riemann surface has a neighborhood that is conformally equivalent to either a punctured disc, in which case we say that the boundary component is a puncture, or an annulus, in which case we say that it is a hole. Two topologically finite Riemann surfaces have the same analytic type if there is a homeomorphism between them that preserves both holes and punctures. A topologically finite Riemann surface is analytically finite if it has no holes.

Theorem A. Let $S$ be a topologically finite Riemann surface of genus $g>0$. Then there is a closed Riemann surface $\bar{S}$ of genus $g$, and there is a conformal embedding $f: S \rightarrow \bar{S}$ so that $f(S)$ is a circle domain on $\bar{S}$. This representation is unique; that is, if there is another closed Riemann surface $\bar{S}^{\prime}$, also of genus $g$, and there is a conformal embedding $f^{\prime}: S \rightarrow \bar{S}^{\prime}$, so that $f^{\prime}(S)$ is also a circle domain, then there is a conformal homeomorphism $h: \bar{S} \rightarrow \bar{S}^{\prime}$ with $f^{\prime}=h \circ f$.

We have already remarked that the existence part of the above theorem is due to Haas $[\mathrm{H}]$; we outline his proof in $\S 4$.

\section{TeICHMÜLLER AND MODULI SPACES}

In this section we outline, using Fuchsian groups, the real analytic version of Bers' development of Teichmüller space and Bers fiber space, together with their modular groups [B].

Let $S$ be a hyperbolic Riemann surface of finite toplogical type and positive genus $g$. Represent $S$ as $\mathbf{H}^{2} / F$, where $F$ is a finitely generated Fuchsian group, normalized so that 0,1 , and $\infty$ are fixed points of hyperbolic elements of $F$. Note that the punctures correspond to parabolic elements of $F$, while the elements of $F$ corresponding to holes are hyperbolic.

For our purposes, a deformation of $F$ is a monomorphism $\phi: F \rightarrow$ $\operatorname{PSL}(2, \mathbf{R})$ (the group of orientation preserving isometrics of $\mathbf{H}^{2}$ ) with the following property. There is an orientation preserving (quasiconformal) homeomorphism $f$ of the closed upper half-plane onto itself so that $\phi$ is induced by 
$f$; that is, $f \circ g(z)=\phi(g) \circ f(z)$ for all $g \in F$, and for all $z \in \mathbf{H}^{2}$. This requirement implies that the induced homeomorphism between $S=\mathbf{H}^{2} / F$ and $\mathbf{H}^{2} / \phi(F)$ preserves both holes and punctures.

A deformation $\phi$ is normalized if the associated homeomorphism $f$ fixes 0 , 1 , and $\infty$.

The set of all normalized deformations of $F$, denoted by $T(F)$, is an open subset of the homomorphism space, $\operatorname{Hom}(F, \operatorname{PSL}(2, \mathbf{R}))$ modulo conjugation, and as such is a real analytic manifold. If $F$ is of the first kind (this is equivalent to the statement that $\mathbf{H}^{2} / F$ has no holes), then $T(F)$ is (real analytically equivalent to) the usual Teichmüller space $T(S)$. Also, if $F$ is of the second kind, then we can identify $T(F)$ with the reduced Teichmüller space of Earle [E].

A deformation $\psi$ of $F$, with the property that $\psi(F)=F$, is called a modular deformation. The group of all modular deformations, $\hat{M}=\hat{M}(F)$, is called the super modular group. It acts on $T(F)$ by right multiplication; that is, for each modular deformation $\psi$, and for each element $\phi$ of $T(F)$, there is an element $A=A(\phi, \psi)$ of $\operatorname{PSL}(2, \mathbf{R})$ so that $A_{*}\left(\phi \circ \psi^{-1}\right)$ is a normalized deformation, where $A_{*}$ denotes conjugation by $A$. We set $\psi(\phi)=A_{*}(\phi \circ$ $\left.\psi^{-1}\right)$. The transformation $A$ depends real analytically on both $\phi \in T(F)$ and $\psi \in \hat{M}(F)$. The super modular group also acts on the Bers fiber space $V(F)=T(F) \times \mathbf{H}^{2}$ by $\psi[\phi, z]=\left[A_{*}\left(\phi \circ \psi^{-1}\right), A(z)\right]$.

For any group $G$ acting on a space $X$, the kernel, $K$, of the action is the (normal) subgroup acting as the identity, and the effective part is $H=G / K$. The Modular group, $M_{T}(F)$, is the effective part of the action of $\hat{M}$ on $T(F)$, and the modular group, $m_{V}(F)$, is the effective part of the action on $V(F)$. The moduli space, $R(F)=T(F) / M_{T}(F)$ can be identified with the space of conformally distinct Riemann surfaces of the same analytic type as $S=\mathbf{H}^{2} / F$.

The Bers space $B(F)=V(F) / m_{V}(F)$ is a fiber space with base $R(F)$, where the fiber over the point $S^{\prime} \in R(F)$ is the surface $S^{\prime}$ factored by its conformal automorphism group. One can also view $B(F)$ as being the space of positive divisors of degree one on Riemann surfaces having the same analytic type as $S$.

\section{KOEBE GROUPS}

A Kleinian group $G$ is a discrete group of Möbius transformations that acts discontinuously somewhere on the extended complex plane. The set of points at which $G$ acts discontinuously is called the regular set and is denoted by $\Omega=\Omega(G)$. Its complement, the limit set, is denoted by $\Lambda=\Lambda(G)$. The connected components of $\Omega$ are called components of $G$.

In everything that follows, we will be concerned only with torsion-free groups; we will assume, without further mention, that all Kleinian groups are torsion-free. 
An isomorphism $\phi$ between Kleinian groups is called type-preserving if both $\phi$ and $\phi^{-1}$ preserve parabolic elements.

A function group is a Kleinian group $G$ with an invariant component $\Delta_{0}$ (that is, $g\left(\Delta_{0}\right)=\Delta_{0}$ for all $g \in G$ ), where $S_{0}=\Delta_{0} / G$ is analytically finite.

A Kleinian group is geometrically finite if it has a finite sided fundamental polyhedron for its action on hyperbolic 3-space; equivalent definitions can be found in [M2, Chapter VI].

A Koebe group is a geometrically finite function group with invariant component $\Delta_{0}$, where every component other than $\Delta_{0}$ is a circular disc.

We remark that the class of Koebe groups includes the finitely generated Fuchsian groups and the Euclidean groups.

The following existence and uniqueness theorems for Koebe groups were proven in [M1].

Theorem 1. Let $G$ be a function group with invariant component $\Delta_{0}$. Then there is a Koebe group $\tilde{G}$, with invariant component $\tilde{\Delta}_{0}$, and there is a conformal homeomorphism $f: \Delta_{0} \rightarrow \tilde{\Delta}_{0}$, where $f$ induces a type-preserving isomorphism of $G$ onto $\tilde{G}$.

Theorem 2. Let $G_{1}$, with invariant component $\Delta_{1}$, and $G_{2}$, with invariant component $\Delta_{2}$, be Koebe groups. Suppose there is a conformal homeomorphism $f: \Delta_{1} \rightarrow \Delta_{2}$, where $f$ induces a type-preserving isomorphism of $G_{1}$ onto $G_{2}$. Then $f$ is the restriction of a Möbius transformation.

Let $F$ be a Fuchsian or Euclidean group, acting on $X$, where $X$ is either $\mathbf{H}^{2}$ or $\mathbf{C}$, and let $S$ be a circle domain on $X / F$. Note that $X / F$ is closed of genus $g ; g=1$, and $F$ is purely parabolic if and only if $X=\mathbf{C} ; g>1$, and $F$ is of the first kind and purely loxodromic (actually hyperbolic) if and only if $X=\mathbf{H}^{2}$. In any case, $F$ is finitely generated.

Let $A$ denote the preimage, under the natural projection, of $S$ in $X$; then $A$ is a subdomain of $X$ with the following properties: $A$ is $F$-invariant; every connected component of $X-A$ is either a point or a circular disc; and $A / F$ is a topologically finite Riemann surface. Such a domain $A$ is called a circle domain for $F$.

Let $A$ be a circle domain for the Fuchsian or Euclidean group $F$. For each circle $C_{j}$ on the boundary of $A$, other than the limit circle of $F$, let $r_{j}$ denote reflection in $C_{j}$. Let $\tilde{F}$ denote the group generated by $F$ and all the $r_{j}$, and let $\hat{F}$ be the orientation preserving half of $\tilde{F}$.

Lemma 1. $\hat{F}$ is a Koebe group. Further, if $F$ is Fuchsian, then $\hat{F}$ is purely loxodromic, while if $F$ is Euclidean, then every parabolic element of $\hat{F}$ is conjugate in $\tilde{F}$ to an element of $F$.

Proof. Since $S=A / F$ is topologically finite, we can easily find a fundamental domain $D$ for $F$, where the boundary of $D$ does not meet any of the $C_{j}$. 
Inside $D$, there are only finitely many of the $C_{j}$; relabel the $C_{j}$ so that these are $C_{1}, \ldots, C_{k}$. Then $\tilde{F}$ is generated by $F$, together with $r_{1}, \ldots, r_{k}$.

Euclidean groups and finitely generated Fuchsian groups are geometrically finite, and it is almost immediate that $G=\left\langle r_{1}, \ldots, r_{k}\right\rangle$, the group generated by $r_{1}, \ldots, r_{k}$, is also geometrically finite. Draw a loop $W$ in the interior of $D$, where $W$ separates $C_{1} \cup \cdots \cup C_{k}$ from $\partial D$. Then $W$ divides the extended complex plane into two closed discs, one of which is precisely invariant under the identity in $F$, and the other is precisely invariant under the identity in $G$. (A set $X$ is precisely invariant under the subgroup $H \subset G$ if $X$ is $H$-invariant, and $g(X) \cap X=\varnothing$ for all $g \in G-H$.) By the first combination theorem [M2, p. 149], $\tilde{F}$ is geometrically finite. The combination theorem also shows that if $F$ is Euclidean, then $\tilde{F}$ has no noninvariant components, while if $F$ is Fuchsian, then every noninvariant component of $\tilde{F}$ is conjugate in $\tilde{F}$ to the lower half-plane, and thus is a circular disc.

We continue with our use of the combination theorem and note that every elliptic or parabolic element of $\tilde{F}$ is conjugate to an element in either $F$ or $G$. Since the circles defining the reflections that generated $G$ are all disjoint, every torsion element of $G$ is a reflection; hence $\hat{F}$ is torsion-free. For the same reason, $G$ contains no parabolics, and $F$ contains parabolics if and only if $F$ is Euclidean. Hence $\hat{F}$ is purely loxodromic if $F$ is Fuchsian. If $F$ is Eulcidean, then every parabolic element of $\tilde{F}$, hence also of $\hat{F}$, is conjugate to an element of $F$.

The existence of an invariant component for $\hat{F}$ is essentially immediate. Since $\tilde{F}$ has an invariant component, so does its subgroup $\hat{F}$.

Since $\hat{F}$ is of finite index in $\tilde{F}$, it is also geometrically finite, and of course, every noninvariant component of $\hat{F}$ is also a circular disc; we have shown that $\hat{F}$ is a Koebe group.

\section{Proof of Theorem A}

We start with the outline of the proof of existence.

We are given a topologically finite Riemann surface $S$ of genus $g \geq 1$. For each possible such topological type, choose a base surface $S_{0}$, and choose a Fuchsian group $H_{0}$, so that $\mathbf{H}^{2} / H_{0}=S_{0}$. Then there is a deformation $\phi$ of $H_{0}$, so that $\mathbf{H}^{2} / \phi\left(H_{0}\right)=S$. Write $H=\phi\left(H_{0}\right)$. Let $\tilde{S}$ be the double of $S$; that is, we consider $H$ as a Kleinian group, let $\Omega(H)$ be its set of discontinuity, and let $\tilde{S}=\Omega(H) / H$. Each of the arcs of discontinuity of $H$ on the extended real axis projects to a simple closed geodesic (in the Poincare metric) on $\tilde{S}$, and there are finitely many such geodesics; call them $w_{1}, \ldots, w_{k}$. Let $v_{1}, \ldots, v_{l}$ be disjoint small loops about each of the punctures of $\tilde{S}$. Let $p: X \rightarrow \tilde{S}$ be the highest regular covering of $\tilde{S}$ for which each of the loops $w_{j}, j=1, \ldots, k$ and each of the loops $v_{m}, m=1, \ldots, l$ lifts to a loop. Then [M1] we can choose $X$ to be a plane domain, where the group of deck transformations is a Koebe group, $\tilde{G}$, and $X=\Delta_{0}$ is the invariant component of $\tilde{G}$. 
There is a natural orientation reversing conformal homeomorphism $j$ of $\tilde{S}$ which as $\bigcup w_{m}$ as its fixed point set. Let $J$ be some lift of $j$ to $\Delta_{0}$, where $J$ keeps some component of the preimage of some $w_{m}$ pointwise fixed. Then, denoting the map $z \rightarrow \bar{z}$ by $*$, we see that $J \circ *$ is a conformal homeomorphism mapping the invariant component $\Delta_{0}$ of $\tilde{G}$ onto the invariant component of $* \tilde{G} *$, and $J \circ *$ induces a type-preserving isomorphism of $\tilde{G}$ onto $* \tilde{G} *$. We conclude from Theorem 2 that $J$ is an orientation reversing fractional linear transformation; it follows that its fixed point set is a circle.

Next let $A$ be some connected component of the preimage of $S$ in $\Omega$, and let $G=\operatorname{Stab}(A)$ be its stabilizer in $\tilde{G}$. Then $A$ is $G$-invariant, $A / G$ is a surface of finite topological type, and $A$ is bounded by limit points of $G$, circles and points. Since $\tilde{G}$ is a Koebe group, $G$ is either Fuchsian or Euclidean, and $A$ is a circle domain for $G$.

We turn now to uniqueness. Suppose that we have two such representations: $S=A_{1} / G_{1}=A_{2} / G_{2}$. Then there is a conformal homeomorphism $f: A_{1} \rightarrow A_{2}$, where $f$ conjugates $G_{1}$ onto $G_{2}$. Since $G_{1}$ (or $\left.G_{2}\right)$ contains parabolic elements if and only if $S$ has genus 1 , in which case $G_{1}$ is purely parabolic, the induced isomorphism between $G_{1}$ and $G_{2}$ is type-preserving. Since $f$ conjugates $G_{1}$ onto $G_{2}$, it is clear that $f$ maps the circular boundary components of $A_{1}$ onto those of $A_{2}$. For $m=1,2$, we form the group $\tilde{G}_{m}$, generated by $G_{m}$ and the reflections in all the boundary circles of $A_{m}$. Let $\hat{G}_{m}$ be the orientation preserving half of $\tilde{G}_{m}$. One easily sees that $\tilde{G}_{m}$, and so also $\hat{G}_{m}$, has an invariant component $\hat{\Delta}_{m}$ of its set of discontinuity. It follows from the reflection principle that $f$ extends to a conformal homeomorphism, which we call by the same name, $f: \hat{\Delta}_{1} \rightarrow \hat{\Delta}_{2}$. This extended map $f$ conjugates $\tilde{G}_{1}$ onto $\tilde{G}_{2}$, and so also conjugates $\hat{G}_{1}$ onto $\hat{G}_{2}$.

It follows from Lemma 1 both that $\hat{G}_{m}$ is a Koebe group, and that $f$ induces a type-preserving isomorphism of $\hat{G}_{1}$ onto $\hat{G}_{2}$.

It now follows from Theorem 2 that $f$ is the restriction of a fractional linear transformation. Since $f$ conjugates $G_{1}$ onto $G_{2}$, we have shown that $\bar{S}$ is unique up to conformal equivalence.

By the above uniqueness statement, we can assume that $G_{1}=G_{2}=G$, and that $f$ is a fractional linear transformation conjugating $G$ onto itself; that is, $f$ lies in the normalizer of $G$ (in the appropriate space of all orientation preserving isometries). Then $f$ projects to a conformal homeomorphism of $\bar{S}$ which maps $A_{1} / G_{1}$ onto $A_{2} / G_{2}$. This concludes the proof of Theorem A.

\section{REMARKS}

1. Theorem A assigns a unique nonnegative number to each boundary component of a topologically finite Riemann surface $S$ of positive genus. This number is 0 if the boundary component is a puncture; otherwise, it is the radius of the hole when $S$ is represented as a circle domain. While the center of the removed 
disc, on the closed surface $\bar{S}$, is only determined up to a conformal automorphism of $\bar{S}$, the radius is well defined, for every conformal automorphism is an isometry in the canonical metric.

2. In the case that $S$ has only one boundary component, then Theorem A says that $\bar{S}$ is unique, the radius of the hole is unique, and the center of the hole is uniquely determined on $\bar{S} / \Gamma$, where $\Gamma$ is the conformal automorphism group of $\bar{S}$.

In the special case of genus 1 , with one boundary component, then we can locate the center of the hole at the projection of the origin.

3. Theorem A is somewhat unsatisfactory when $S$ has more than one boundary component. One example of the difficulty occurs when the automorphism group of $\bar{S}$ is cyclic of order 4 . Let $\gamma$ be a generator of the automorphism group, and let $z$ be a point on $\bar{S}$ with the property that $z_{0}=z, z_{1}=\gamma(z), z_{2}=\gamma^{2}(z)$, and $z_{3}=\gamma^{3}(z)$ are all distinct. For sufficiently small $\alpha$, the disc of radius $\alpha$ about each of these points, in the canonical metric, is a topological disc, and these discs are all disjoint. Let $U_{m}$ be the disc of radius $\alpha$ about $z_{m}$. Then $\bar{S}-\left(U_{1} \cup U_{2}\right)$ is conformally distinct from $\bar{S}-\left(U_{1} \cup U_{3}\right)$. However on the surface $\bar{S} / \Gamma$, these two surfaces appear the same.

\section{AN APPLICATION TO MODULI SPACES}

For the remainder of this paper, we restrict our attention to the case of surfaces with exactly one boundary component.

Let $S$ be a surface with one boundary component, and genus $g>0$. Then there is a Fuchsian or Euclidean group $F$ so that $S=\mathbf{H}^{2} / F$. Theorem A describes a mapping from the moduli space $R(F)$ into a certain space, which we now describe. We first take up the case that $g=1$. Let $\bar{S}$ be the closed torus into which we have a canonical circle embedding of $S$. Then there is a Euclidean group $G$, with unit minimal translation length, so that $S=A / G$, where $A$ is the complement in $\mathbf{C}$ of a $G$-invariant set of circular discs, centered at the origin and its translates. Then $S$ is completely described by a point $\tau \in \mathbf{H}^{2} / \operatorname{PSL}(2, \mathbf{Z})$, and the radius $r$. Since $G$ has unit minimal translation length, $0 \leq r<\frac{1}{2}$. We have shown that for $g=1$, Theorem A defines a bijection $\Phi: R(F) \rightarrow \mathbf{H}^{2} / \operatorname{PSL}(2, \mathbf{Z}) \times\left[0, \frac{1}{2}\right)$.

If $g>1$, then we can represent $S$ as $A / G$, where $G$ is a Fuchsian group acting on $\mathbf{H}^{2}$, and $A$ is a $G$-invariant open subset of $\mathbf{H}^{2}$, whose complement in $\mathbf{H}^{2}$ is a set of circular discs of hyperbolic radius $r$. The center is however only defined on $\mathbf{H}^{2} / \Gamma$, where $\Gamma$ is the normalizer of $G$. In this case, Theorem A defines a mapping $\Phi$ from $R(F)$ into (not onto) $B(G) \times[0, \infty)$.

Lemma 2. The map $\Phi$ is real analytic, except perhaps at $r=0$.

Proof. We follow the above outline of the construction of $A$ and $G$. Start with the surface $S$; then construct its double; then uniformize its double by a 
particular Koebe group; then $G$ is a subgroup of this Koebe group, which is well defined up to conjugation. The map from $S$ to its double is real analytic, and it is a standard application of the Ahlfors-Bers solution of the Beltrami equation [A-B] that the map from the finite doubled surface to the Koebe group is real analytic (see [M1]). Since the subgroup $G$ depends real analytically on $S$, so does the surface $\bar{S}=\mathbf{H}^{2} / G$. For genus 1 , observe that the Koebe group, after normalization, is generated by $g_{1}(z)=z+1, g_{2}(z)=z+\tau$, $g_{3}(z)=z /\left(\sigma_{1} z+1\right)$, and $g_{4}(z)=z /\left(\sigma_{2} z+1\right)$. We can choose these generators so that reflection in the circle of radius $r$ conjugates $g_{1}$ into $g_{3}$, and conjugates $g_{2}$ into $g_{4}$. It is then an easy computation to see that the radius depends real analytically on $\tau, \sigma_{1}$, and $\sigma_{2}$. For $g>1$, the Koebe group is the free product of two Fuchsian groups, and there is a reflection that conjugates one of these groups into the other. Normalize so that one of the two groups acts on the unit disc, and so that the other acts on a disc of Euclidean radius $\rho^{2}<1$, centered at the origin; i.e., the reflection circle has radius $\rho$. Then the hyperbolic radius of the reflection circle (inside the unit disc) is $r=\frac{1}{2} \log (1+\rho) /(1-\rho)$. Since the Koebe group, as a subgroup of $\operatorname{PSL}(2, C)$, depends real analytically on the surface $S$, so does the radius $r$.

The proof that $\Phi^{-1}$ is also real analytic, except perhaps at $r=0$, can be obtained by reversing the above proof.

Theorem B. Let $S$ be a Riemann surface of genus $g>0$ with one hole, and let $R(S)$ be its moduli space. (i) If $g=1$, then $R(S)$ is real analytically equivalent to the product $\mathbf{H}^{2} / \operatorname{PSL}(2, \mathbf{C}) \times\left(0, \frac{1}{2}\right)$. (ii) If $g>1$, then $R(S)$ is topologically equivalent to the product $B(\bar{S}) \times \mathbf{R}$.

Proof. We have already taken care of part (i). For part (ii), we already know that $R(S)$ is topologically equivalent to a fiber space with base $B(\bar{S})$, where the fiber over a point $x$ is the interval $(0, r(x))$, where $r(x)$ is the hyperbolic radius of the largest open embedded disc centered at some preimage of $x$ in $\bar{S}$. An equivalent definition is that $r(x)$ is equal to the injectivity radius at $x$. One can also define it as the radius of the largest open disc contained in the Dirichlet region for $\bar{S}$, centered at $x$. One easily sees that $r(x)$ is a continuous function of the point $x$ in $B(\bar{S})$.

In conclusion, we remark that, if we fix the surface, and let the point vary, then the function $r(x)$ need not be differentiable at a point $x$ where the boundary of the largest embedded disc touches itself four or more times.

\section{REFERENCES}

[A-B] L. V. Ahlfors and L. Bers, Riemann's mapping theorem for variable metrics, Ann. of Math. 72 (1960), 385-404.

[B] L. Bers, Fiber spaces over Teichmüller spaces, Acta Math. 130 (1973), 89-126.

[E] C. J. Earle, Reduced Teichmüller spaces, Trans. Amer. Math. Soc. 126 (1967), 54-63. 
[G] H Grötzsch, Die Werte des Doppelverhältnisses bei schlichter konformer Abbildung, Sitzungsber. Preuss Akad. Wiss. Phys.-Math. Kl. (1933).

[H] A. Haas, Linearization and mappings onto pseudocircle domains, Trans. Amer. Math. Soc. 282 (1984), 415-429.

[M1] B. Maskit, On the classification of Kleinian groups: I. Koebe groups, Acta Math. 135 (1975), 249-270.

[M2] _ Kleinian groups, Springer-Verlag, Berlin, 1988.

[R] H. L. Royden, The interpolation problem for schlicht functions, Ann. of Math. 60 (1954), 326-344.

[S] K. Strebel, Ein Klassifizierungsproblem für Riemannsche Flächen vom Geschlecht 1., Arch. Math. 48 (1987), 77-81.

State University of New York at Stony Brook, Stony Brook, New York 11794-3651 